\title{
Relationship Between 2-Dimensional Frontal Plane Measures and the Knee Abduction Angle During the Drop Vertical Jump
}

\author{
Brad W. Willis, Katie Hocker, Swithin Razu, Aaron D. Gray, Marjorie Skubic, Seth L. Sherman, \\ Samantha Kurkowski, and Trent M. Guess
}

\begin{abstract}
Context: Knee abduction angle (KAA), as measured by 3-dimensional marker-based motion capture systems during jumplanding tasks, has been correlated with an elevated risk of anterior cruciate ligament injury in females. Due to the high cost and inefficiency of KAA measurement with marker-based motion capture, surrogate 2-dimensional frontal plane measures have gained attention for injury risk screening. The knee-to-ankle separation ratio (KASR) and medial knee position (MKP) have been suggested as potential frontal plane surrogate measures to the KAA, but investigations into their relationship to the KAA during a bilateral drop vertical jump task are limited. Objective: To investigate the relationship between KASR and MKP to the KAA during initial contact of the bilateral drop vertical jump. Design: Descriptive. Setting: Biomechanics laboratory. Participants: A total of 18 healthy female participants (mean age: 24.1 [3.88] y, mass: 65.18 [10.34] kg, and height: 1.63 [0.06] m). Intervention: Participants completed 5 successful drop vertical jump trials measured by a Vicon marker-based motion capture system and 2 AMTI force plates. Main Outcome Measure: For each jump, KAA of the tibia relative to the femur was measured at initial contact along with the KASR and MKP calculated from planar joint center data. The coefficient of determination $\left(r^{2}\right)$ was used to examine the relationship between the KASR and MKP to KAA. Results: A strong linear relationship was observed between MKP and KAA $\left(r^{2}=.71\right)$, as well as between KASR and KAA $\left(r^{2}=.72\right)$. Conclusions: Two-dimensional frontal plane measures show strong relationships to the KAA during the bilateral drop vertical jump.
\end{abstract}

Keywords: injury risk screening, motion capture, knee-to-ankle separation ratio, medial knee position

Each year an estimated 38,000 anterior cruciate ligament (ACL) injuries are sustained by females in the United States with a 34\% increase in ACL reconstruction rates between 2002 and 2014. ${ }^{1}$ Unfortunately, those suffering from an ACL injury require longterm rehabilitation with a higher risk for reinjury and demonstrate increased rates of knee pain and prevalence of osteoarthritis. ${ }^{1-4}$ Investigations into preventive screening techniques monitoring ACL injury risk factors aimed at mitigating female injury rates are warranted. ${ }^{1,2}$

The use of 3-dimensional marker-based motion capture systems in conjunction with force platforms has long been the "gold standard" for determining kinematic and kinetic biomechanical parameters. $^{3,4}$ Altered lower-extremity kinematic and kinetic parameters demonstrated by females during dynamic jumping tasks have been extensively investigated to assess potential correlations to ACL injury risk. ${ }^{3-5}$ Specifically, an excessive knee abduction angle (KAA), a measure of tibia displacement relative to the femur acquired by 3-dimensional motion capture systems, has been linked to elevated risk of injury to the ACL. ${ }^{3}$ A bilateral jumplanding task, the drop vertical jump (DVJ), has been utilized to obtain KAA measures correlated to ACL injury risk. ${ }^{3}$ Moreover, the initial contact phase of the DVJ has been suggested as espe-

Willis and Guess are with the Department of Physical Therapy, School of Health Professions, University of Missouri, Columbia, MO, USA. Hocker is with the School of Medicine, University of Missouri, Columbia, MO, USA. Razu and Kurkowski are with the College of Engineering/Biological Engineering, University of Missouri, Columbia, MO, USA. Gray, Sherman, and Guess are with the Department of Orthopedic Surgery, University of Missouri, Columbia, MO, USA. Skubic is with the College of Engineering/Electrical Engineering and Computer Science, University of Missouri, Columbia, MO, USA. Willis (willisbw@health. missouri.edu) is corresponding author. cially relevant due to elevated torque between the femur and tibia during periods of limited knee flexion at landing, stressing the ACL. ${ }^{2}$ Interestingly, most 2-dimensional frontal plane measures assess the peak flexion phase of the DVJ exclusively, neglecting the increased ACL stress during the initial contact phase. ${ }^{2,4,5}$

Unfortunately, 3-dimensional motion capture systems are expensive, space prohibitive, and require advanced training to operate, precluding widespread usage. ${ }^{5,6}$ Consequently, investigations into simplified surrogate 2-dimensional measures to monitor knee valgus collapse during motions such as the DVJ have garnered attention in recent years. ${ }^{5,6}$ The knee-to-ankle separation ratio (KASR), a bilateral lower-extremity measure defined as the frontal plane distance between the knee joint centers divided by the frontal plane distance between the ankle joint centers, has been suggested. ${ }^{5,6}$ Mizner et $\mathrm{al}^{5}$ highlighted the KASR as a potential surrogate to KAA, but demonstrated only a weak to moderate relationship $\left(r^{2}=.25\right)$ to the KAA at peak flexion of the DVJ. In this study, Mizner et $\mathrm{al}^{5}$ only examined peak knee flexion of the DVJ, excluding analysis at initial contact.

The medial knee position (MKP) has also been associated with an increased risk of ACL injury during a bilateral DVJ task. ${ }^{4}$ The MKP is produced for each limb, being defined as the perpendicular distance from the knee center to a line connecting the hip and ankle in the frontal plane. ${ }^{4}$ Medial knee displacement is the difference in MKP from a neutral standing position to the MKP during peak flexion during the DVJ, demonstrating greatest predictive value for recurrent ACL tears. ${ }^{4}$ Unfortunately, analyses of MKP at initial DVJ contact are lacking. ${ }^{4}$ Examining the relationship of MKP to KAA at initial contact of the bilateral DVJ is especially valuable due to the high stress placed on the ACL at this time point and its simplified determination compared with the KAA. ${ }^{2}$ 
The purpose of this study was to investigate the relationship between KAA and KASR, as well as KAA and MKP during DVJ initial contact in female participants. Our hypothesis is that KASR and MKP would demonstrate a moderate to strong relationship to the KAA during the bilateral DVJ at initial contact.

\section{Methods}

\section{Design}

Investigators utilized a descriptive study design within a university biomechanics laboratory.

\section{Participants}

With institutional review board approval at the University of Missouri, we recruited 18 healthy female participants (mean age: 24.1 [3.88] y, mass: 65.18 [10.34] kg, height: 1.63 [0.06] m). All participants provided informed consent prior to involvement. Exclusion criteria included history of neurological illness or lowerextremity injury within 12 months.

\section{Procedures}

Anthropometric measurements assessed included body mass, height, interanterior iliac spine distance, leg length, knee width, and ankle width for both limbs. According to the lower body Plugin-Gait model, 16 skin-surface retroreflective markers were placed on the trunk and legs. ${ }^{7}$ Four additional markers were placed on the medial epicondyle of each knee and medial malleolus of each ankle to estimate thigh rotation offset, shank rotation offset, and tibial torsion during a static stance. Motion data were captured using an 8-camera Vicon MX-T40S retroreflective motion capture system (Vicon Motion Systems Ltd, Oxford, United Kingdom) with a sample rate of $100 \mathrm{~Hz}$. The kinematic data were filtered using a fourth-order zero-lag, low-pass Butterworth filter with a 6-Hz cutoff and subsequently processed using Vicon ${ }^{\mathrm{TM}}$ Nexus software.

Verbal instructions and demonstration of the DVJ were given to each subject prior to recording the motion. Participants stood on a $31-\mathrm{cm}$ high platform while wearing their own athletic shoes, dropped from the platform to the ground, and immediately performed a maximal vertical jump, as if going up to get a rebound in basketball. ${ }^{3}$ One practice trial was completed, and 5 successful trials per subject were recorded. If a participant jumped instead of dropping off the box or if submaximal effort was displayed, she was asked to repeat the jump. ${ }^{4}$ Participants wore shoes and dropped onto dual force platforms (Optima; Advanced Mechanical Technology, Inc, Watertown, MA) with each foot landing on a separate platform. Initial contact with the ground after dropping from the box was determined from the force platforms using a threshold of $10 \mathrm{~N}$.

The KAA for each limb along with the joint centers for the hips, knees, and ankles were recorded at initial contact. Joint center data were obtained from the Vicon Plug-in-Gait skeletal model and used to calculate the KASR and the MKP. Only joint center coordinates in the frontal plane were used in the 2-dimensional KASR and MKP calculations (Figures 1 and 2).

\section{Statistical Analyses}

As the KASR is a bilateral lower-extremity measure, the KAA for the left and right lower-extremity were averaged for each jump trial for KASR comparison. Once this was completed for each trial, the average across 5 successful trials (ensemble average) was

compared between the KASR and the KAA. This resulted in a total of 18 comparisons. Alternatively, the MKP is a unilateral lowerextremity measure, allowing for direct comparison to the right and left KAA. Subsequently, the ensemble average of MKP for the

Eq. 1

$$
\operatorname{KASR}=\frac{(\overline{\text { Knee }})}{(\overline{\text { Ankle }})}
$$

Eq. 2

$$
\begin{gathered}
M K P=\frac{2 \sqrt{S *(S-\overline{\mathbf{H K}}) *(\mathrm{~S}-\overline{\mathrm{KA}}) *(\mathrm{~S}-\overline{\mathrm{HA}})}}{\overline{\mathbf{H A}}} \\
\mathrm{S}=(\overline{\mathrm{HK}}+\overline{\mathrm{KA}}+\overline{\mathrm{HA}}) / 2
\end{gathered}
$$

Figure 1 - Equations for frontal plane measures. $\overline{\text { Ankle indicates the }}$ frontal plane distance from the left ankle to the right ankle joint centers; $\overline{\mathrm{HA}}$, the frontal plane distance from the hip to the ankle joint centers; $\overline{\mathrm{HK}}$, the frontal plane distance from the hip to the knee joint centers; $\overline{\mathrm{KA}}$, the frontal plane distance from the knee to the ankle joint centers; KASR, knee-to-ankle separation ratio; $\overline{\mathrm{Knee}}$, the frontal plane distance from the left knee to the right knee joint centers; MKP, medial knee position. If the knee center is lateral to the line connecting the hip and ankle, MKP is positive. If the knee center is medial to the line connecting the hip and ankle, MKP is negative.
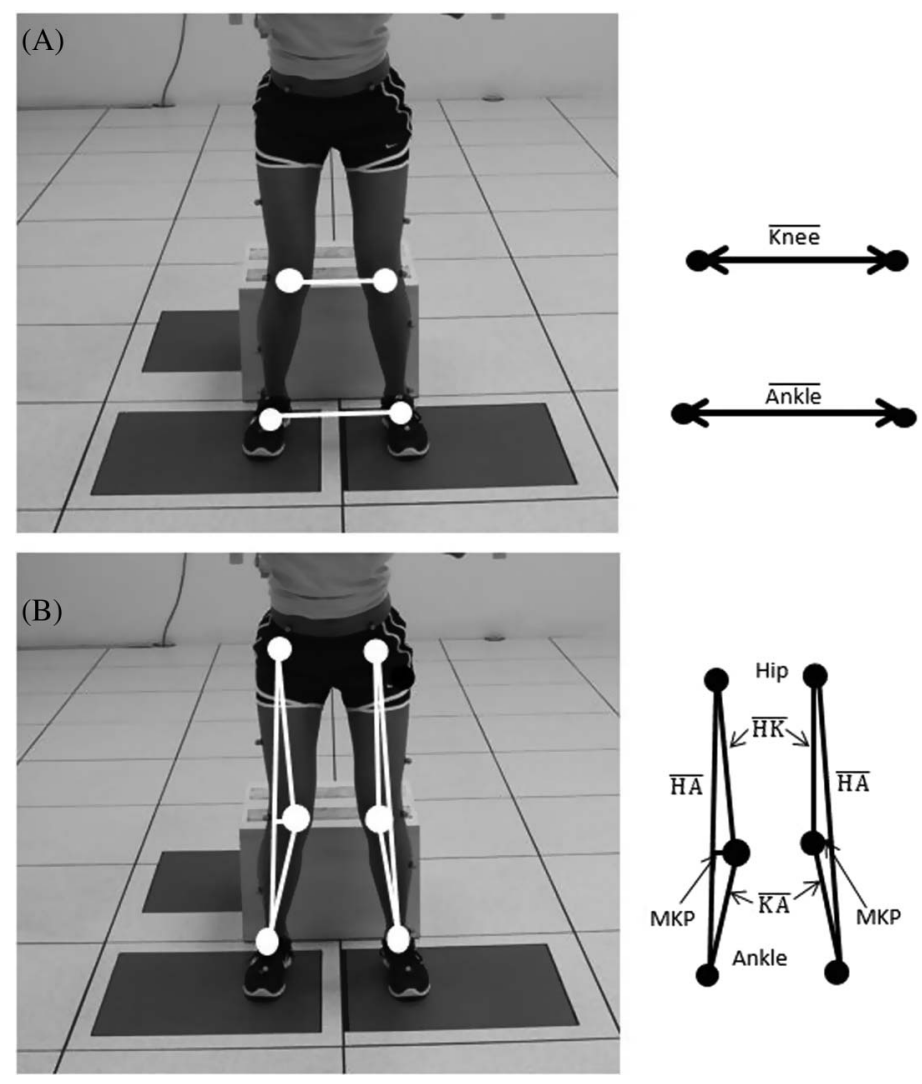

Figure 2 - Visualization of frontal plane measures at initial drop vertical jump contact. (A) KASR and (B) MKP. Ankle indicates the frontal plane distance from the left ankle to the right ankle joint centers; $\overline{\mathrm{HA}}$, the frontal plane distance from the hip to the ankle joint centers; $\overline{\mathrm{HK}}$, the frontal plane distance from the hip to the knee joint centers; $\overline{\mathrm{KA}}$, the frontal plane distance from the knee to the ankle joint centers; Knee, the frontal plane distance from the left knee to the right knee joint centers; MKP, medial knee position. 
right lower-extremity was compared with the ensemble average for the right KAA during the bilateral DVJ. Concurrently during the bilateral DVJ, the ensemble average of the left MKP was compared with the ensemble average of the left KAA. This resulted in a total of 36 comparisons when examining the relationship between the MKP and KAA for the right and left lower-extremities. The coefficient of determination $\left(r^{2}\right)$ between KAA and KASR and between KAA and MKP was calculated using Microsoft Excel 2013 (Microsoft; Redmond, WA). A coefficient of determination greater than .64 was classified as a strong linear relationship, and a coefficient of determination between .26 and .64 was considered a moderate linear relationship. ${ }^{8}$ Furthermore, to determine if significant differences $(P<.05)$ existed between limbs in KAA or MKP measurements, paired $t$ tests were performed.

\section{Results}

A strong linear relationship was observed between MKP and KAA $\left(r^{2}=.71\right)$, as well as between KASR and KAA $\left(r^{2}=.72\right)$ (Figure 3). Ensemble averages of MKP for left and right lower-extremity were 14.48 (16.04) and 11.61 (13.44) $\mathrm{mm}$, respectively. Ensemble averages of KAA for left and right lower-extremity were $1.38^{\circ}$ $\left(4.98^{\circ}\right)$ and $1.04^{\circ}\left(3.78^{\circ}\right)$, respectively. Ensemble average of KAA between the right and left limb per trial was $1.21^{\circ}\left(4.36^{\circ}\right)$, with an ensemble average KASR of 0.80 (0.8). No significant differences were seen between left and right KAA or MKP values at initial contact of the DVJ.
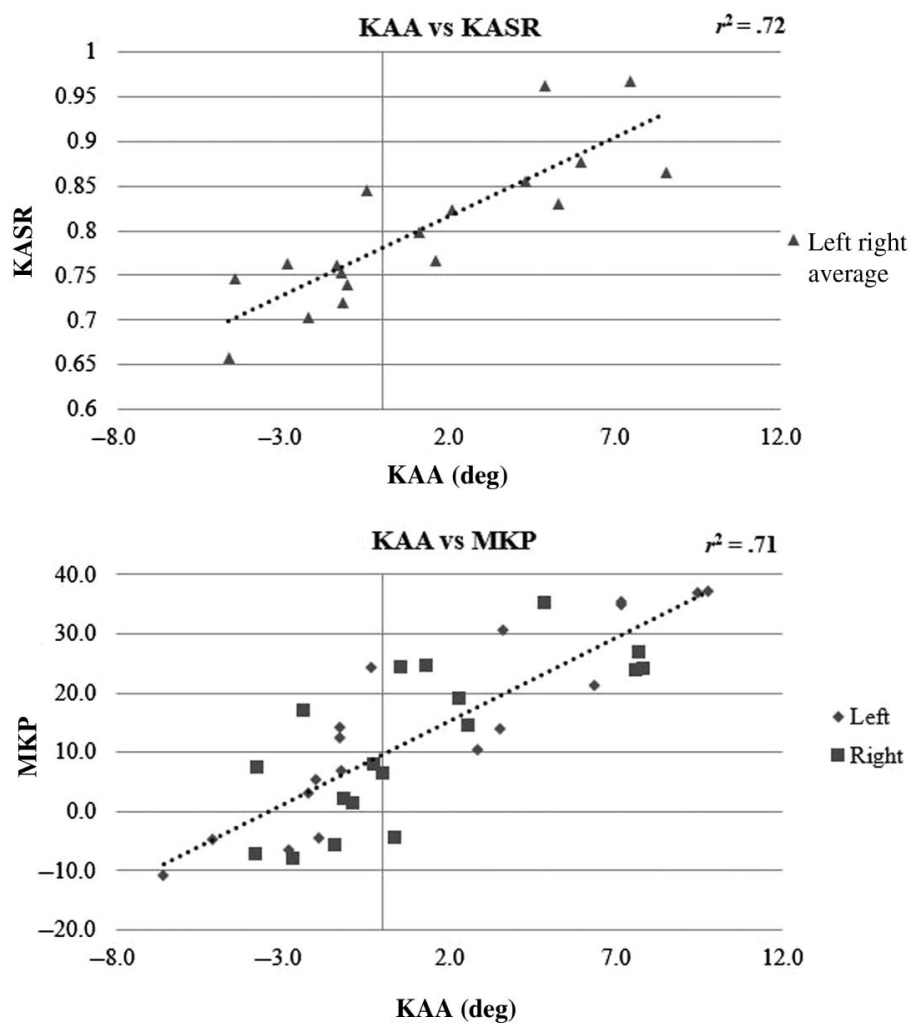

Figure 3 - Relationship between the KAA and frontal plane measures at initial drop vertical jump contact. (A) Linear regression model of KAA versus KASR. (B) Linear regression model of KAA versus MKP. KAA indicates knee abduction angle; KASR, knee-to-ankle separation ratio; MKP, medial knee position.

\section{Discussion}

The KAA has been associated with an increased risk for ACL injury, as well as other ligamentous injuries to the knee. ${ }^{2,3}$ This study found that at initial contact of the bilateral DVJ, the frontal plane KASR and MKP measures had a strong linear relationship to the KAA. Such frontal plane surrogate measures of KAA, based on frontal plane joint center locations by 3-dimensional skeletal models, offer promise for cost-effective and simplified methods in assessing knee valgus collapse. Notably, investigations using infrared depth cameras have shown potential of translating such 2-dimensional surrogates from 3-dimensional skeletal models, such as the KASR, into clinically useful data with more portable and efficient systems. ${ }^{6}$ Particularly, the markerless Microsoft Kinect V2 depth camera has been highlighted as a valuable tool to provide efficient and clinically useful frontal plane estimations of the hip, knee, and ankle joint centers during dynamic jumplanding tasks. ${ }^{6}$

Although both MKP and KASR were found to be strongly correlated with KAA resulting from the bilateral DVJ, MKP provides a unilateral estimate of frontal plane knee displacement that may have greater clinical importance. Specifically, the MKP offers a unilateral estimate of frontal plane mechanics, as opposed to the bilateral KASR. Even though this study examined the relationship between the MKP and KAA using the ensemble averages of the right and left lower-extremities, as no significant differences between limbs were seen, the authors recommend examining the relationship of the MKP to KAA during a unilateral DVJ or similar single-limb landing task. This is especially valuable as an increased risk for noncontact lower-extremity injury is often associated with altered unilateral landing mechanics. ${ }^{3,4}$ Furthermore, a bilateral DVJ will distribute vertical ground reaction forces between both extremities, likely resulting in a reduced amount of MKP displacement at IC compared with a unilateral landing assessment. Alternately, the KASR is a simpler measurement and easier to produce than the MKP, as the required estimation of the hip joint center can be more challenging than locating centers of the knee and ankle joint. ${ }^{9}$ Nevertheless, both frontal plane measures, KASR and MKP, offer promise for screening ACL injury risk in females as a 2-dimensional surrogate of KAA during bilateral DVJ initial contact.

Despite the fact that this investigation does not utilize portable sensor technology, we believe this study provides significant clinical relevance. By advancing the understanding of how frontal plane measures generated by 3 -dimensional motion capture systems correlate to the KAA, investigators may identify the selection of more promising frontal plane surrogates for future investigations. This notion is supported by Cesar et al, ${ }^{10}$ who concluded that investigation using 3-dimensional motion analysis to generate correlations of 2-dimensional frontal measures is a first step to screen for aberrant movement patterns. Moreover, advances in depth camera sensors appear promising to address the space, cost, and technical limitations of traditional gait laboratories. 6

With the frontal plane MKP and KASR demonstrating a strong relationship to the KAA, this study reinforces the potential value of surrogate 2-dimensional measures in assessing movements linked to risk of ACL injury. As innovations in injury prevention strategies and mobile technology continue, 2-dimensional lower-extremity frontal plane assessments during dynamic movement tasks offer potential for simple, efficient, and evidencedbased screening. 


\section{References}

1. Herzog MM, Marshall SW, Lund JL, Pate V, Spang JT. Incidence of anterior cruciate ligament reconstruction among adolescent females in the United States, 2002 through 2014. JAMA Pediatr. 2017;171(8): 808-810. PubMed ID: 28604937 doi:10.1001/jamapediatrics. 2017.0740

2. Bates NA, Nesbitt RJ, Shearn JT, Myer GD, Hewett TE. Knee abduction affects greater magnitude of change in ACL and MCL strains than matched internal tibial rotation in vitro. Clin Orthop Relat Res. 2017;475(10):2385-2396. PubMed ID: 28455730 doi:10.1007/ s11999-017-5367-9

3. Hewett TE, Myer GD, Ford KR, et al. Biomechanical measures of neuromuscular control and valgus loading of the knee predict anterior cruciate ligament injury risk in female athletes: a prospective study. Am J Sports Med. 2005;33(4):492-501. PubMed ID: 15722287 doi:10.1177/0363546504269591

4. Krosshaug T, Steffen K, Kristianslund E, et al. The vertical drop jump is a poor screening test for ACL injuries in female elite soccer and handball players: a prospective cohort study of 710 athletes. Am J Sports Med. 2016;44(4):874-883. PubMed ID: 26867936 doi:10. $1177 / 0363546515625048$
5. Mizner RL, Chmielewski TL, Toepke JJ, Tofte KB. Comparison of 2-dimensional measurement techniques for predicting knee angle and moment during a drop vertical jump. Clin J Sport Med. 2012;22(3):221-227. PubMed ID: 22544058 doi:10.1097/JSM. 0b013e31823a46ce

6. Gray AD, Willis BW, Skubic M, et al. Development and validation of a portable and inexpensive tool to measure the drop vertical jump using the Microsoft Kinect ${ }^{\mathrm{TM}}$ V2. Sports Health. 2017;9(6):537-544. PubMed ID: 28846505 doi:10.1177/1941738117726323

7. Davis RB III, Öunpuu S, Tyburski D, Gage JR. A gait analysis data collection and reduction technique. Hum Mov Sci. 1991;10(5): 575-587. doi:10.1016/0167-9457(91)90046-Z

8. Moore DS, McCabe GP, Craig BA. Introduction to the Practice of Statistics. 6th ed. New York, NY: W.H. Freeman \& Co.

9. Kainz H, Carty CP, Modenese L, Boyd RN, Lloyd DG. Estimation of the hip joint centre in human motion analysis: a systematic review. Clin Biomech. 2015;30(4):319-329. PubMed ID: 25753697 doi:10. 1016/j.clinbiomech.2015.02.005

10. Cesar GM, Pfeifer CM, Burnfield JM. 3-dimensional versus 2-dimensional comparison of knee valgus collapse during vertical jump: clinical implications for ACL risk of injury assessment. J Sports Med Ther. 2017;2:32-38. doi:10.29328/journal.jsmt.1001006 